\title{
Review: tissue adhesives are as effective as standard wound closure for cosmetic outcomes of traumatic lacerations
}

\author{
Farion $K$, Osmond MH, Hartling L, et al. Tissue adhesives for traumatic lacerations in children and adults. Cochrane \\ Database Syst Rev 2002;(3):CD003326 (latest version 28 May 2002).

\section{QUESTION: Are tissue adhesives (TAs) as effective as standard wound closure (SWC) for traumatic lacerations in children and adults? Are some TAs better than others?}

\section{Data sources}

Published and unpublished studies in any language were identified by searching the Cochrane Controlled Trials Register (CD ROM 2001 Issue 4), the Cochrane Wounds Group Specialized Trials Register (November 2001), Medline (1966 to October 1, 2001), and EMBASE/ Excerpta Medica (1988 to September 1, 2001); reviewing the bibliographies of retrieved articles; and by contacting study authors and manufacturers of TAs

\section{Study selection}

Randomised controlled trials were included if they compared TAs with SWC (ie, sutures, staples, or adhesive strips) or compared 2 different TAs for acute, linear lacerations that were $<12$ hours old resulting from blunt or sharp trauma in patients seen in an emergency department (ED) or primary care setting. Exclusion criteria were stellate lacerations, puncture wounds, or mammalian bites; wounds that were infected, heavily contaminated, or devitalised; wounds crossing joints or mucocutaneous junctions, or in hair bearing areas; or wounds in patients with keloid formation or chronic illness that could impair healing.

\section{Data extraction}

One reviewer extracted data on study design, randomisation method, withdrawals/dropouts, funding source, participant age, wound characteristics, type of adhesive, wound preparation and dressing, comparison intervention, outcome measures, and results. Study quality was assessed using the 5 point Jadad scale. Main outcome was cosmetic appearance, primarily assessed using the Cosmetic Visual Analogue Scale (CVAS) or the Wound Evaluation Score (WES). Other outcomes included pain during the procedure, procedure time, and complications (infection, dehiscence, erythema, and discharge).

Alberta Heritage

Foundation for Medical

Research, Canada

For correspondence: Dr K Farion,

Emergency Medicine,

Departments of

Pediatrics and

Medicine, University of

Ottawa, Children's

Hospital of Eastern

Ontario, Ottawa,

Ontario, Canada.

farion@cheo.on.ca

\section{Main results}

9 trials met the selection criteria. 8 trials compared a TA (butylcyanoacrylate or octylcyanoacrylate) with SWC, and 1 trial compared 2 different TAs. TAs did not differ from SWC for cosmetic appearance at 1-3 months or 9-12 months assessed using the CVAS (weighted mean difference [WMD] $0.6,95 \% \mathrm{CI}-5.0$ to $6.3,6$ trials; and $4.3, \mathrm{CI}-5.8$ to 14,2 trials, respectively) or WES (odds ratio 0.97 , CI 0.58 to $1.62,4$ trials; and 1.36 , CI 0.62 to 2.95, 2 trials, respectively). Patients who were treated

Tissue adhesives (TAs) v standard wound closure (SWC) for traumatic lacerations in adults and children*

\begin{tabular}{|c|c|c|c|c|c|}
\hline \multirow[b]{2}{*}{ Complications } & \multirow[b]{2}{*}{ Number of trials ( $n$ ) } & \multicolumn{2}{|c|}{$\begin{array}{l}\text { Weighted event } \\
\text { rates }\end{array}$} & \multirow[b]{2}{*}{ RRI (95\% CI) } & \multirow[b]{2}{*}{ NNH (Cl) } \\
\hline & & TAs & swc & & \\
\hline \multirow[t]{2}{*}{ Dehiscence } & 7 trials $(n=650)$ & $6.6 \%$ & $2.8 \%$ & $125 \%$ (8 to 365$)$ & 27 (15 to 200 ) \\
\hline & & & & $\operatorname{RRR}(\mathrm{Cl})$ & NNT (Cl) \\
\hline Erythema & 2 trials $(n=186)$ & $14 \%$ & $25 \%$ & $44 \%$ (2 to 69 ) & 9 (5 to 200$)$ \\
\hline
\end{tabular}

with TAs had lower pain scores reported by patients, parents, physicians, or nurses (range of WMDs in scores -15.7 to -10.8$)$. Procedure time was 5.7 minutes shorter with TAs (CI 3.1 to 8.2, 5 trials). TAs were associated with higher rates of dehiscence than SWC, but lower rates of erythema (table). The groups did not differ for infections, delayed closure, or discharge. One study compared butylcyanoacrylate with octylcyanoacrylate and found no differences in cosmetic appearance, pain, procedure time, dehiscence, or infection.

\section{Conclusion}

In adults and children with traumatic lacerations, tissue adhesives are as effective as standard wound closure for cosmetic appearance and reduce procedural pain and time, but increase dehiscence rates.

\section{COMMENTARY}

The systematic review by Farion et al examined whether TAs are as effective as SWC for traumatic lacerations in children and adults. Meta-analysis showed that the main outcome, cosmetic appearance at 1-3 months and 9-12 months, was similar for patients treated with TAs and those treated with SWC. Meta-analysis also showed that TAs reduced procedure related pain and reduced procedure time by about 5 minutes. The review found a small, but statistically significant, increase of $4 \%$ in wound dehiscence after treatment with TAs. Further research is needed to identify the specific characteristics of wounds that can and cannot be effectively managed with TAs. For example, the review excluded wounds that were infected, heavily contaminated, or devitalised, those crossing joints or mucocutaneous junctions, and those in hair bearing areas. Although the review also sought to compare the effectiveness of different TAs, only 1 trial compared 2 different TAs and found no difference for any outcome.

Limitations of the review included the poor methodological quality of the included studies (1-3 points out of 5 on the Jadad scale). Because of the nature of the intervention, none of the studies could blind patients. This could have affected patient reports of pain.

The primary advantage of TAs for treatment of lacerations in the ED is the reduction in procedure related pain. The reduction in procedure time has the added benefit of reducing the stress of ED visits. 6 of the studies included in the analysis were limited to paediatric patients. The benefits of reduced pain and procedure time will be particularly relevant to paediatric patients, who are likely to be frightened and anxious about suturing. System advantages include a faster turn around in high volume, congested EDs. As well, TAs do not require a follow up visit for removal as do SWC techniques. Given the small increased risk for wound dehiscence, patients should be carefully assessed to determine the potential for dehiscence before deciding to use TAs.

Glenn Donnelly, RN, MN

Assistant Professor, College of Nursing, University of Saskatchewan, Regina, Saskatchewan, Canada Nurse, Emergency Department, Pasqua Hospital Saskatoon, Saskatchewan, Canada

*Abbreviations defined in glossary; RRI, RRR, NNH, NNT, and $\mathrm{Cl}$ calculated from data in article. 